\title{
Zur Kenntnis der organischen Gerüstsubstanz des Anthozoënskeletts.
}

I. Mitteilung.

Von

Car1 Th. Morner, Upsala.

(Der Redaktion zugegangen am 28, Januar 1907.)

Einleitung.

Bei mehreren der zur Klasse der Anthozoën gerechneten Tierformen kommen wohlentwiekelte Skelettbildungen vor. Es ist die in diesen enthaltene organische Gerüstsubstanz, die Gegenstand der vorliegenden Untersuchung bỉldet. Zur Orientierung dürfte es angebracht sein, mit einer kurzen Übensicht über die Systematik der Anthozoön und ihre Skelettbildung za beginnen.

Die Klasse Anthozoa ${ }^{1}$ ) umafaßt zwei,Ordnungen; Alcyo:aria (= Octactinia Ehrbg) und Zoantharia (= Polyactinia Ehrbg ex parte).

Alcyonaria zerfällt wieder nach einer seit langem verwendeten und immer moch beibehaltenen Einteilung (siehe $\boldsymbol{u}$. a. Milne Edwards, ${ }^{2}$ ) Studer, ${ }^{3}$ ) v. Koch) ${ }^{4}$ ) in folgende drei Unterordnungen, wenn auch über die gegenseitige Abgrenzung denselben die Ansichten etwas auseinander gegangen sind (in der folgenden Darstellung balten wir uns an die Aufassung v. Kochs).

1) Selbst stellt sie eine Gruppe der Metazoën dar, nach dem Schema :

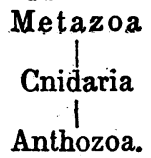

2) [P., S. 102.] 3) [R., S. 9.] 4) [K., S. 16-17.]

Hoppe-Seyler's Zeitschrift f. physiol. Chemie. LI. 


\section{Gorgonacea. \\ II. Pennatulacea. \\ III. Alcyonacea.}

Ein an organischer Gerüstsubstanz reiches Skelett ist bei allen Gorgonaceen und Pennatulaceen, wie auch bei gewissen Alcyonaceen vorhanden.

Von den zu den Zoantharia gehörenden, zahlreichen Unterordnungen besitzt nur Antipathidea ein derartiges Skelett. $\left.{ }^{1}\right)$

Die Frage nach der Entstehung und Entwickelung des Skeletts der Anthozoën hat im Laufe der Zeiten eine verschiedene Beurteilung erfahren. Es ist v. Kochs Verdienst, zuerst in befriedigender Weise dieselbe behandelt $\mathrm{zu}$ haben. $\mathrm{Er}$ hat klar nachgewiesen, ${ }^{2}$ ) daß es zwei grundverschiedene Typen gibt: ektodermales (Ekto-)Skelett und mesodermales (Meso-)Skelett. Das Ektoskelett wird von ektodermalen (epithelialen) Zellen abgesondert und wird stets in zusammenhängender Schicht abgesetzt. Befindet sich nun die sezernierende Epithelschicht auf der Außenseite der Polypenmasse (sehr selten vorkommend), so bildet das Skelett eine schützende Hülle, und es wird als Hüllskelett bezeichnet. Wird das Skelett dagegen von einem nach innen wachsenden Epithel ausgeschieden, sodaß es als Stütze oder Achse fungiert, so heißt es Achsenskelett (auch bezeichnet: "Axis» oder "Sclerobase»). Das Ektoskelett, sowohl Hüll- als Achsenskelett, zeigt konzentrische Schichtung und ist ausnahmslos reich an organischer Gerüstsubstanz. Das Mesoskelett hat seinen Ursprung in ganz kleinen, hauptsächlich aus Calciumsalz bestehenden, krystallinischen Körperchen, welche bei manchen Arten zu größeren Massen («Scleriten * oder *Spicula») verschmelzen. Diese Skeletteile sind mit einer oft kaum darstellbaren, bisweilen aber derben organischen Scheide überzogen, welche eine starke Verdickung erfahren kann und dann konzentrische Schichtung und hornartige Festigkeit zeigt. Natür-

1) Die übrigen Anthozoën entbehren entweder eines Skeletts (z. B. die Seeanemonen) oder sind mit Skeletten ausgerüstet, die fast ausschließlich aus anorganischer Substanz (Calciumcarbonat etc.) bestehen (z. B. die Edelkoralle).

2) $[$ K., S. $10-12$. 
lich weist das Mesoskelett nur in letztgenanntem Fall einen größeren Reichtum an organischer Gerüstsubstanz auf. Die bei der hier vorliegenden Untersuchung in Frage kommenden organischen Gerüstsubstanzen rühren bei den Gruppen Gorgonacea, Pennatulacea und Antipathidea von dem Ekto-, genauer dem Achsenskelett, bei den Alcyonaceen von dem Mesoskelett her. ${ }^{1}$ )

Eine systematische, möglichst viele Arten von den verschiedenen Hauptgruppen umfassende Untersuchung der organischen Gerüstsubstanz der Anthozoën hinsichtlich der Art und Menge der darin enthaltenen Haloide schien mir in mehr als einer Hinsicht Interesse zu bieten. Ebenso lag Anlaß vor, dem Gehalt der hierhergehörigen Substanzen an Schwefel eine gewisse Aufmerksamkeit zu widmen.

Das Untersuchungsmaterial (und seine Vorbehandlung).

$\mathrm{Zu}$ dem erforderlichen, verhältnismäßig reichhaltigen Untersuchungsmaterial haben mit größter Bereitwilligkeit Beiträge geleistet die Herren Museums- und Institutsdirektoren Dr. A. Appellöf (Bergen), Dr. M. Henze (Neapel), Dr. L. Jägerskiöld (Göteborg), Prof. G. Levinsen (Kopenhagen), Prof.E. Lönnberg (Stockholm), Cand. Phil. O. Nordgaard (Trondhjem), Prof. Hj. Théel (Stockholm), Prof. T. Tullberg (Upsala) und Lic. Phil. Hj. Östergren (Christineberg), wie auch Herr Dr. G. Swenander (Upsala). Allen diesen Herren fühle ich mich für das freundliche Entgegenkommen, das sie mir bei der Beschaffung des Materials gezeigt haben, zu größtem Dank verpflichtet.

Alles Material wurde einer vorbereitenden Behandlung unterzogen, die darauf ausging, das organische Skelett rein von der Beimengung sonstiger Gewebebestandteile und möglichst frei von anorganischen Stoffen herzustellen. Durch Aufweichen in verdünnter Essigsäure, nachfolgender, erforderlichenfalls wiederholter Behandlung mit harter Bürste und Abspülen wurde die

1) In einer zu den Alcyonaceen gehörenden Familie, den Cornulariden, findet sich die als *Hüllskelett bezeichnete Mesoskelettform vertreten; trotz ernster Bemühungen ist es mir aber nicht gelungen, Untersuchungsmaterial aus der genannten Gruppe zu erhalten. 
Polypenmasse (das Coenenchym.) vollständig entfernt. Das Skelett (mittels Schere, Zange oder in vereinzelten Fällen Drechseln mechanisch zerteilt), ${ }^{1}$ ) wurde dann einer gründlichen Extraktion mit verdünnter Essigsäure + Tołuol bei $+40^{\circ} \mathrm{C}$. unterzogen. Die Säure wurde nach wenigen Tagen gewechselt und damit fortgefahren, bis die Extraktionsflüssigkeit nicht mehr oder nur ganz schwach mit Ammoniumoxalat reagierte. Hierbei zeigten verschiedene Arten ein ganz verschiedenartiges Verhalten. Einige zeigten sich bereits nach $1-2$ Wochen reaktionsfrei, und zwar trat dieses nicht nur bei Arten ein, die schon an sich kalkarm waren (z. B. Paramuricea- und Plexaura-Arten), sondern auch bei solchen, die offenbar stark kalkinkrustiert waren (z. B. Primnoa-Arten). Bei solchem Verlauf der Entkalkung wies das Endprodukt äußerst unbedeutenden Aschengehalt auf (einige Zehntel \%). Andere Arten dagegen (Gorgonella- und LophogorgiaArten, gewisse Pennatulaceen), die auf die gleiche Weise $11 / 2-2$ Monate lang, ja noch länger behandelt wurden, sind dabei doch nicht reaktionsfrei geworden und haben einen etwas höheren Aschengehalt $(2-3 \%)$ beibehalten. Dieser Unterschied beruht der Hauptsache nach nicht auf dem prozentischen Calciumgehalt des Ausgangsmaterials, sondern auf der Art der darin enthaltenen Calciumsalze. Wiederholte Versuche haben gezeigt, daß Calciumphosphat (bisweilen in sehr beträchtlicher Menge vorkommend) und auch Calciumsulfat es sind, die mit solcher Hartnäckigkeit der Einwirkang der verdünnten Essigsäure widerstehen (während Calciumcarbonat ohne Schwierigkeit sich entfernen läßt). Bei zu Kontrollzwecken vorgenommener Behandlung mit verdünnter $\mathrm{HCl}$ (statt Essigsäure) war das Resultat ein ganz anderes: die Entkalkung ging dabei rasch und vollständig vor sich. Da indessen der Hauptzweck des Materiałs der war, als Unterlage für Haloidbestimmungen zu dienen, so habe ich bei der Vorbereitung des Analysenmaterials die sonst

1) Bei den Repräsentanten der Familie Isidae, deren Skelett im Gegensatz zu dem der übrigen Gorgonaceen - aus alternierenden, hornartigen Scheiben und harten Kalksegmenten gebildet ist, warden, vor der weiteren Vorbehandlung, die letzteren auf mechanischem Wege: entfernt. 
weit bequemeren und in gewissen Fällen in bezug auf Entkalkung effektivere $\mathrm{HCl}$ nicht anwenden wollen. Andere Arten endlich nahmen in der genannten Hinsicht eine Mittelstellung ein. Eine solche vorbereitende, sorgfältige Behandlung des Materials ist absolut notwendig, wenn es sich um Haloid- und Sehwefelbestimmungen für die organische Gerüstsubstanz handelt, indem das Skelett - wie ich bei mehreren, eigens zu diesem Zwecke angestellten Versuchen gefunden - in nativer Form regelmäßig und bisweilen in bedeutenden Mengen anorganisches Chlor und ebensolchen Schwefel (Chloride, resp. Sulfate) einsehließt. Gleichwohl ist bei der Mehrzahl der früheren Untersuchungen dieser Umstand unberücksichtigt geblieben, weshalb die Resultate mehr oder weniger haben irreführen müssen.

Nach fortgesetzter Digestion mit destilliertem Wasser, resp. Alkohol und Trocknen an der Luft bei gelinder Wärme wurde das von jeder Art erhaltene Material - nachdem eine Feuchtigkeitsund Aschenbestimmung ausgeführt worden - analysiert. In allen den Fällen, wo nur 1 oder ganz wenige, im ganzen gleichgrobe Exemplare der Art bearbeitet wurden, wurde die gesamte (luftgetrocknete) Ausbeute gewogen, wonach unter Berücksichtigung des Feuchtigkeitsgehalts (und, wo dies geschehen konnte, des Aschengehalts) die totale Ausbeute an anorganischer Gerüstsubstanz festgestellt wurde. Es geschah dies, um ein objektiveres MaB für die Größe der analysierten Exemplare zu erhalten, als es z. B. die bloße Messung der «Länge», «Breite» oder Stammdicke hätte geben können. Zu besonderem Zwecke wurden in einigen Fällen verschiedene Exemplare derselben Art, wie auch verschiedene Partien - gröbere (= ältere) Stämme oder Äste, bezw. feinere (= jüngere) Äste - von demselben Exemplar gesondert behandelt.

\section{Analysenmethoden.}

Je nach der Menge des schließlich erhaltenen, gereinigten Materials konnte die analytische Prüfung mehr oder weniger umfassend angestellt werden. Bei jeder ${ }^{1}$ ) Art, von der - nach erfolgter Feuchtigkeits- und Aschenbestimmung - eine $5 \mathrm{~g}$ 
oder mehr aschenfreier Trockensubstanz entsprechende Menge zurückgeblieben war, wurde folgendes Prüfungsverfahren angewendet.

Eine genau $5 \mathrm{~g}$ aschenfreier Trockensubstanz entsprechende Materialmenge wird auf dem Wasserbad zusammen mit $50 \mathrm{ccm}$ konzentrierter Natronlauge (bereitet durch Auflösen von $500 \mathrm{~g}$ Natrium hydricum e natrio metallico paratum in destilliertem Wasser und Verdünnung bis $\mathrm{zu} 1 \mathrm{l}$ Volumen) erwärmt. Nachdem hierbei, eventuell nach dem Ersatz des verdunsteten Wassers, die Substanz sich aufgelöst hat, wird allmählich weiter erhitzt, bis die Gasentwickelung aufhört und die Schmelze eine fast weiße Farbe annimmt. ${ }^{2}$ ) In diesem Stadium werden ganz kleine Portionen von feinpulverisiertem, reinem $\mathrm{NaNO}_{3}$ bis zur vollständigen Entfärbung der Schmelze zugesetzt. Die Schmelze wird in warmem Wasser gelöst, und die Lösung nach dem $\mathrm{Ab}$ kühlen auf das Volumen von $150 \mathrm{ccm}$ gebracht.

\section{A. Bestimmung von Haloiden.}

$90 \mathrm{ccm}$ der Lösung werden mit destilliertem Wasser verdünnt und stark abgekühlt. Nach Überführung in einen (in kaltes Wasser versenkten) Erlenmeyerschen Kolben werden ganz langsam $40 \mathrm{ccm}$ stark abgekühlte verdünnte $\mathrm{H}_{2} \mathrm{SO}_{4}$ (bereitet aus 1 Volumen konzentrierter Säure + 3 Volumen destilliertem Wasser) zugesetzt. Bei hierbei erreichter saurer Reaktion wird Jod, wenigstens partiell, schon wegen des in der Schmelze vorhandenen (aus dem zugesetzten Nitrat entstandenen) Nitrits frei. In einem geräumigen Scheidetrichter wird geschüttelt, nachdem mit etwas konzentrierter Schwefelsäure, mit $\mathrm{N}_{2} \mathrm{O}_{3}$ gesättigt, sowie mit $\mathrm{CS}_{2}{ }^{1}$ ) (3 Portionen à 30,30 und $15 \mathrm{ccm}$ )

1) Nur bei Nr. 14 konnte dieses Programm nicht durchgeführt werden, da ein Teil des Materials verloren ging. Desgleichen bei Nr. 27 a, dessen (allerdings reichliches) Material für andere Zwecke völlig verbraucht wurde, bevor ich mich zur Anstellung systematischer quantitativer Haloidbestimmungen entschloß.

2) Von Krukenbergs einander widersprechenden Angaben [L, S. 5 bezw. M., S. 1845] über Entstehung von Indol bei Schmelzen mit Alkali ist die positive nach meinem Befunde die richtige. 
versetzt worden ist. Vor dem Schütteln mit der letzten $\mathrm{CS}_{2}$-Portion wird durch Zusatz einiger weiterer Tropfen $\mathrm{N}_{2} \mathrm{O}_{3}$-Schwefelsäure geprüft, ob das Jod schon vollständig frei geworden ist (dies ist überall der Fall gewesen).

a) Die gesammelte Jod-CS - -Lösung wird, nach sorgfältigem Waschen und Zusatz von verdünnter $\mathrm{NaHCO}_{3}$-Lösung, mit $\mathrm{n} / 50 \mathrm{Na}_{2} \mathrm{~S}_{2} \mathrm{O}_{3}$-Lösung titriert. Die daraus berechnete. Jodmenge, dividiert durch 3 , repräsentiert die in $1 \mathrm{~g}$ der Trockensubstanz enthaltene Menge dieses Stoffs.

b) Die Wasserlösung, vermengt mit dem ersten Waschwasser von der $\mathrm{CS}_{2}$-Lösung, wird bis zu $300 \mathrm{ccm}$ Volumen aufgefüllt und filtriert.

a) $200 \mathrm{ccm}$ werden mit gewöhnlicher Salpetersäure (20 ccm) und, unter Umrühren, mit 5\% iger $\mathrm{AgNO}_{3}$-Lösung in gelindem Überschuß versetzt. Das Gewicht des lege artis ausgewaschenen usw. Niederschlages, dividiert durch 2, gibt die Summe von $\mathrm{AgBr}$ und AgCl per $1 \mathrm{~g}$ analysierter Trockensubstanz an.

Aus dem beim Erhitzen in trockenem Gl-Strom entstandenen Gewichtsverlust ergibt sich die in dem Niederschlag enthaltene Menge AgBr, woraus wieder die Menge Brom per $1 \mathrm{~g}$ analysierter Trockensubstanz berechnet werden kann. Die Gewichtsdifferenz zwischen gefundenem $\mathrm{AgBr}+\mathrm{AgCl}$ und $\mathrm{AgBr}$ allein gibt, nach Division durch 2, die Menge AgCl bezw., nach Umrechnung, Chlor in $1 \mathrm{~g}$ Substanz an.

$\mathrm{Da}$ die quantitativen Br-Bestimmungen auf indirekter $\mathrm{Me}-$ thode basieren, so ist großes Gewicht darauf gelegt worden, daß in jedem einzelnen Falle auch mittels einer zuverlässigen qualitativen Prüfungsmethode das Element ad oculos demonstriert wurde.

B) An dem Rest des Filtrats (gegen $100 \mathrm{ccm}$, entsprechend $1 \mathrm{~g}$ analysierter Substanz), versetzt mit ein paar Kubikzentimetern $\mathrm{CS}_{2}$, wird einequalitative Bromprüfung mittels sukzessiven, vorsichtigen Zusetzens von Chlorwasser und Umschütteln zwischen jedem Zusatz angestellt, wobei rotgelbe bis orangerote

1) Durch Schütteln mit metallischem $\mathrm{Hg}$ und Umdestillieren frisch rektifiziert. 
Färbung der $\mathrm{CS}_{2}$-Schicht die Gegenwart von Brom anzeigt. Die Stärke des qualitativen Reaktionsresultates hat stets in guter Übereinstimmung mit dem quantitativen Analysenresultat gestanden.

Durch von Anfang bis zu Ende durchgeführte Blindversuche, wobei als Analysenmaterial notorisch haloidfreie Proteinsubstanz zur Verwendung kam, wurden sämtliche angewandten Reagenzien in bezug auf Haloidfreiheit kontrolliert ( $J$ und $\mathrm{Br}$ fehlten vollständig; von $\mathrm{Cl}$ waren nur unwägbare Spuren vorhanden).

\section{B. Bestimmung des Schwefels.}

$60 \mathrm{ccm}$ von der alkalischen Lösung der Schmelze werden auf bekannte Weise behandelt (Übersättigung mit $\mathrm{HCl}$, wiederholte Abrauchung mit $\mathrm{HCl}$, Aufnahme des Restes in 1\%iger

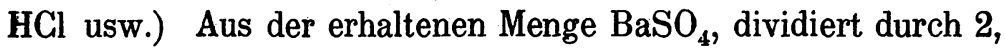
ergibt sich (nach Umrechnen) die Menge Schwefel in $1 \mathrm{~g}$ analysierter Substanz.

Wenn die Menge disponiblen Analysenmaterials (Trockensubstanz) für gewisse Arten mehr oder weniger unter $5 \mathrm{~g}$. betrug, mußten dementsprechende Einschränkungen im Umfang der Analyse vorgenommen werden. In jedem solchen Fall wurde die S-Bestimmung weggelassen. Betrug die Materialquantität nicht einmal $2^{1 / 2} \mathrm{~g}$, so unterblieb auch die quantitative $\mathrm{Br}$ - und Cl-Bestimmung. Dagegen wurden - wegen der Empfindlichkeit der betreffenden Methoden - quantitative J-Bestimmung und qualitative Br-Prüfung in jedem Fall vorgenommen, sogar wenn die vorhandene Materialquantität weniger als $1 \mathrm{~g}$ betrug.

Die quantitativen Werte für Haloide und Schwefel finden sich in der Tabelle unten (S. 46-49) angegeben, berechnet in Prozenten derTrockensubstanz, wo Aschenbestimmung stattgefunden, in Prozenten aschenfreier Trockensubstanz. Daß die Berechnung für aschenfreie Substanz - wegen der Knappheit des Materials - nicht durchgehends hat geschehen können, dürfte hier von keiner oder nur geringer Bedeutung sein, da der Aschengehalt infolge der energischen Vorbehandlung des Untersuchungsmaterials doch jedenfalls nur als verhältnismäßig unbedeutend (höchstens einige wenige Prozente) angesehen werden kann. 
Die bisher veröffentlichten chemischen Untersuchungen über das organische Skelett der Anthozoën beziehen sich so. gut wie ausschlieBlich auf die Gruppe Gorgonacea, ${ }^{1}$ ) welcher Umstand im Verein mit dem anderen, daß mein Untersuchungsmaterial von Gorgonaceen verhältnismäßig am reichhaltigsten gewesen, mich veranlaßt, in erster Linie diese Gruppe zu behandeln.

\section{Gorgonacea. ${ }^{2}$ )}

A. Vorkommen von Haloiden (in organischer Bindung).

\section{Literaturübersicht.}

a) Über das Vorkommen von Jod.

Die Gerüstsubstanz, die in dem Achsenskelett der Gorgonaceen enthalten ist - früher Cornein (cornéine), ${ }^{3}$ ) später Gorgonin ${ }^{4}$ ) genannt - hat schon seit den 1850er Jahren in chemischer Hinsicht Beachtung gefunden. Einen höheren Grad von Interesse gewann diese Substanz indessen erst durch Drechsels Entdeckung 1896 eines sehr beträchtlichen J-Gehalts in dem von ihm bearbeiteten Material (von Gorgonia Cavolini v. Koch). ${ }^{5}$ ) Der J-Gehalt - berechnet aus dem Gewichtsverlust des erhaltenen Silberniederschlages im Cl-Strom - wird als 7,79\% in aschehaltiger Trockensubstanz (mit 7,09\% Asche) angegeben. Daß der J-Gehalt des Gorgonins von Valenciennes und Fremy (beider Arbeiten stammen aus den

1) Überhaupt findet sich in der bisherigen Literatur - überdies außerordentlich flüchtig - nur eine einzige Pennatulacee, und eine vereinzelte Repräsentantin für Antipathidea (dagegen keine Alcyonacee) erwähnt.

2) Die Gorgonaceen werden auch Gorgoniden, Gorgonien oder, mit nicht lateinischem Namen, Hornkorallen genannt.

3) Valenciennes [S., S. 11].

4) Drechsel [D., S. 92].

5) Daß der seit langem bekannte J-Gehalt der Schwämme(Spongien) der Hauptsache nach nicht von Jodiden herrührt, sondern organisch gebunden in der Gerüstsubstanz enthalten ist, war schon früher von Vogel [T., S. 226] nachgewiesen worden (nachdem das J-Vorkommen selbst bereits 1837 von Proust bemerkt worden war).

Die von Drechsel angewandte und später oft zitierte Schreibweise Cavolinii ist nicht richtig. Vgl. die Originalstelle: $\mathbf{}$. Koch [K., S. 58]. 
1850er Jahren) nicht bemerkt worden ist, dazu ist ja kaum etwas zu sagen, daß aber auch Krukenberg (in den 1880er Jahren) denselben ganz übersehen, kann wunderlicher erscheinen, besonders da er mehrere Hydrolysenversuche angestellt hat, u. a. auch an Material von der G. Cavolini nahestehenden, ebenso J-reichen $G$. verrucosa ${ }^{1}$ ) und ferner Elementaranalysen ausgeführt hat, aus denen er sogar eine bestimmte (haloid- und schwefelfreie!) Formel: $\mathrm{C}_{30} \mathrm{~N}_{9} \mathrm{H}_{44} \mathrm{O}_{13}{ }^{2}$ ) abgeleitet hat. Drechsel stellte ferner durch Hydrolyse des erwähnten Gorgonins mittels Baryumhydroxydlösung ein J-reiches Produkt - Jodgorgosäure - dar, das er für eine Aminojodbuttersäure hielt. Später (1903) hat Henze durch Analysieren eines aus demselben Material und auf ähnliche Weise dargestellten J-reichen Spaltungsprodukts $(57,32 \% \mathrm{~J}$ und $3,78 \% \mathrm{~N}$ enthaltend) klar nachgewiesen, daß Drechsels Auffassung der Jodgorgosäure als einer einfachen Säure der Fettreihe nicht aufrechterhalten werden kann. Vielmehr läge hier eine einen aromatischen Komplex enthaltende Säure vor. Mit lobenswerter Vorsicht verzichtet Henze darauf, auf die wegen der Knappheit des Untersuchungsmaterials wenigen analytischen Data eine bestimmte Formel zu gründen. Daß jedoch Henze die Frage in die richtige Bahn geleitet hat, ergibt sich aus Wheeler und Jamiesons ${ }^{3}$ ) 2 Jahre später veröffentlichter Arbeit über Synthese der Jodgorgosäure. Allem nach zu urteilen, haben diese Forscher gezeigt, daß die Natur derselben als 3,5-Dijodtyrosin nachgewiesen:

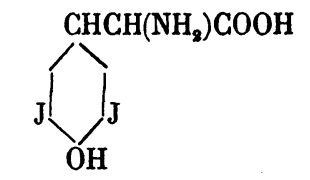

(Berechneter J-Gehalt $58,66 \%$ )

$($, $\mathrm{N}-$, $3,23 \%$,

ein Analogon zu dem von Gorup-Besanez [X] bereits in den 1860 er Jahren synthetisch dargestellten und beschriebenen Di-

1) [L., S. 5.]

2) [M., S. 1846.]

s) [U.] 
bromtyrosin darstellend. In den letzteren Jahren sind quantitative J-Bestimmungen an einer Anzahl anderer Gorgonaceen von den amerikanischen Forschern Mendel (1901) und Cook (1905) publiziert worden. Mendel [0], der dieselbe Bestimmungsmethode wie Drechsel angewandt, hat Material von 3. Arten untersucht. ${ }^{1}$ ) Die angegebenen Werte beziehen sich auf lufttrockenes und offenbar auch stark aschehaltiges Material, indem nichts von vorgenommener Dekalzinierung erwähnt wird (Angaben über Aschebestimmungen fehlen).

Eine reichhaltigere Untersuchungsserie (10 Arten umfassend) ${ }^{2}$ ) ist von Cook [B] geliefert worden. Die Bestimmungen sind teils mit der von Drechsel verwendeten, teils und hauptsächlich mittels der Andrewschen Methode (Titrierung mit $\mathrm{KJO}_{3}$-Lösung bei Gegenwart von $\mathrm{HCl}$ in starkem Überschuß) ausgeführt worden $[\mathrm{A}]$. Das Untersuchungsmaterial ist, wie die hohen Zahlen (5,09 bis hinauf zu 40,37\%) für den Aschegehalt zeigen, nicht dekalziniert worden.

B) Über das Vorkommen von Brom.

Betreffs $\mathrm{Br}$ als konstituirenden Bestandteils der organischen Skelettsubstanz der Gorgonaceen (oder anderer Anthozoën) ${ }^{\text {s) }}$; findet sich in der ganzen Literatur keine einzige positive Angabe. In Drechsels und Henzes Arbeiten wird nichts erwähnt, was darauf hindeutet, daß nach diesem Element ge-

1) 1. Plexaura flexuosa, 2. Gorgonia acerosa und 3. Rhipidigorgia flabellum, sämtlich von Westindien. (Die letztgenannte Art wird in Cooks wie auch in Mendels Arbeit unter dem Namen Gorgonia flabellum aufgeführt; bei Krukenberg u. a. hat sich die fehlerhafte Schreibweise Rhipidogorgia eingeschlichen.)

2) 1. Muricea hebes und 2. M. muricata; 3. Eunicea Rousseaui; 4. Plexaura flexuosa (3 St. Specimina, a-c); 5. Plexaurella crassa; 6. Gorgonia acerosa; 7. Leptogorgia rigida und 8. L. virgulata (2 St. Specimina, a-b); 9. Rhipidigorgia flabellum und 10. Eugorgia aurantiaca.

Fundorte. Bermuda: 2, 3, $4 \mathrm{a}$ und $\mathrm{b}, 5,6$ und 9; Panama: 1 und 7; Westindien: 4c; N. Carolina: $8 \mathrm{a}$; Florida: $8 \mathrm{~b}$; Kalifornien: 10.

8) Dagegen ist es durch Hun deshagens Untersuchungen [I., S. 473] bekannt, daß wenigstens gewisse Spongien neben reichlichen J-Mengen $(8-14 \%), \mathrm{Br}$ (und $\mathrm{Cl}$ ) in augenfälliger Menge (zusammen 1-2\%) enthalten - alles in organischer Bindung (ein Umstand, der offenbar von Cohnheim [C., S. 122] übersehen worden ist). 
sucht, oder daß es gar beobachtet worden wäre. Loew ${ }^{1}$ ) scbeint zwar an die Möglichkeit des Vorkommens von $\mathrm{Br}$ im Gorgonin gedacht zu haben, indem er im Zusammenhang mit der Erwähnung von Drechsels Analysenresultaten ein Fragezeichen bezüglich eines möglichen Gehalts an $\mathrm{Br}$ eingeschoben hat: «Drechsel calls this protein gorgonin. On decomposition it yielded not only iodine, but also 2 per cent chlorine (or bromine?)». Die im Anschluß an diese Bemerkung später ausgeführten Untersuchungen haben indessen, wie es scheinen könnte, zu dem alleraugenscheinlichsten negativen Resultat geführt. So sagt Mendel: ${ }^{2}$ ) "Loew has called attention to the possibility of the presence of bromine in conjunction with the iodine and chlorine in the Gorgonias. With a view to ascertaining this point I have examined very carefully a relatively large quantity $(30 \mathrm{~g})$ of the skeleton substance of Gorgonia acerosa... The results were entirely negative; not a trace of bromine was found». Cooks Untersuchungsresultate an Material von zehn Arten $^{3}$ ) laufen auf das Gleiche hinaus: "In harmony with Professor Mendels observations, bromine could not be detected in either skeleton or coenenchyma».4)

r) Über das Vorkommen von Chlor.

Ein sehr beträchtlicher Gehalt dieses Elements im Gorgonaceenskelett ${ }^{5}$ ) ist von Drechsel ${ }^{6}$ ) für Gorgonia Cavolini $\left(2,18 \%\right.$ ) und von Mendel ${ }^{7}$ ) für Plexaura flexuosa, Gorgonia acerosa und Rhipidigorgia flabellum $(0,86,3,17$ bezw. 1,24\%) angegeben worden. Die Bestimmungen - an nativem Achsenskelett, d. h. Material ausgeführt, das nicht durch Vorbehandlung von anorganischen Stoffen befreit worden war - gründen sich alle auf Erhitzung des erhaltenen Haloidsilberniederschlags im Cl-Strom.

1) [N., S. 21.]

2) [0., S. 245.]

s) Siehe die Anm. 2, S. 43.

4) [B., S. 98.]

5) Betreffs $\mathrm{Cl}$ in der Gerüstsubstanz der Spongien siehe die Anm. 3 auf S. 43 .

ө) $[$ D., S. 97.] 7) [0., S. 245.] 
Utber die organische Gerüstsubstanz des Anthozoënskeletts. I.

2. Eigene Untersuchungen.

a) Einleitung.

Die Anregung $\mathrm{zu}$ diesen Untersuchangen gab mir eine Beobachtung, die ich vor mehr als 10 Jahren machte. An dem von meinem Freunde, nunmehr Professor E. Lönnberg, der von einer Forschungsreise in Florida u. a. eine Reihe Gorgonaceen heimgebracht hatte, mir gütigst überlassenem Material (einem Exemplar von Gorgonia setosa) erhielt ich - bei Versuchen, Drechsels kurz vorher veröffentlichte Angabe über das Vorkommen von $\boldsymbol{J}$ zu bestätigen - unzweideutige Reaktion auch für Brom. Den direkten Anlaß dazu, die Frage nach dem Vorkommen von $\mathrm{Br}$ im organischen skelett der Gorgonaceen (und der Anthozoën liberhaupt) wieder aufzunehmen, gaben mir Mendels und Cooks oben angeführte negative Resultate in Verbindung mit dem Umstande, daß ich gelegentlich neuerdings ausgeführter Wasseranalysen mit den sinnreichen Methoden Bekanntschaft machte, die eine genaue Bestimmung der drei Haloide (J, Br und $\mathrm{Cl}$ ) bei gleichzeitiger Gegenwart ermöglichen. Eine Reihe anderer naheliegender Fragen fand bei der Untersuchung gleichzeitig Berücksichtigung. Bei der Beschaffung des Untersuchungsmaterials wurde natürlich besonders danach gestrebt, die früher (von Drechsel, Mendel und Cook) untersuchten Arten möglichst gut repräsentiert zu erhalten.

b) Siehe Tabelle Seite 46-49.

c) Übersicht über die Resultate (nebst kritischer Prüfung früherer Angaben.)

a) Über das Vorkommen von Jod.

Dieses Element hat sich, wie aus den früheren Untersuchungen auf dem Gebiete zu erwarten war, als bei jeder untersuchten Art konstant vorkommend erwiesen. Die erhaltenen Werte liegen innerhalb weiter Grenzen: 0,05-6,92\%.

Die bisher ausgeführten quantitativen J-Bestimmungen sind - infolge des Umstandes, daß die gleichzeitige Gegenwart von $\mathrm{Br}$ (siehe unten) völlig übersehen worden ist - alle, ohne Aus- 


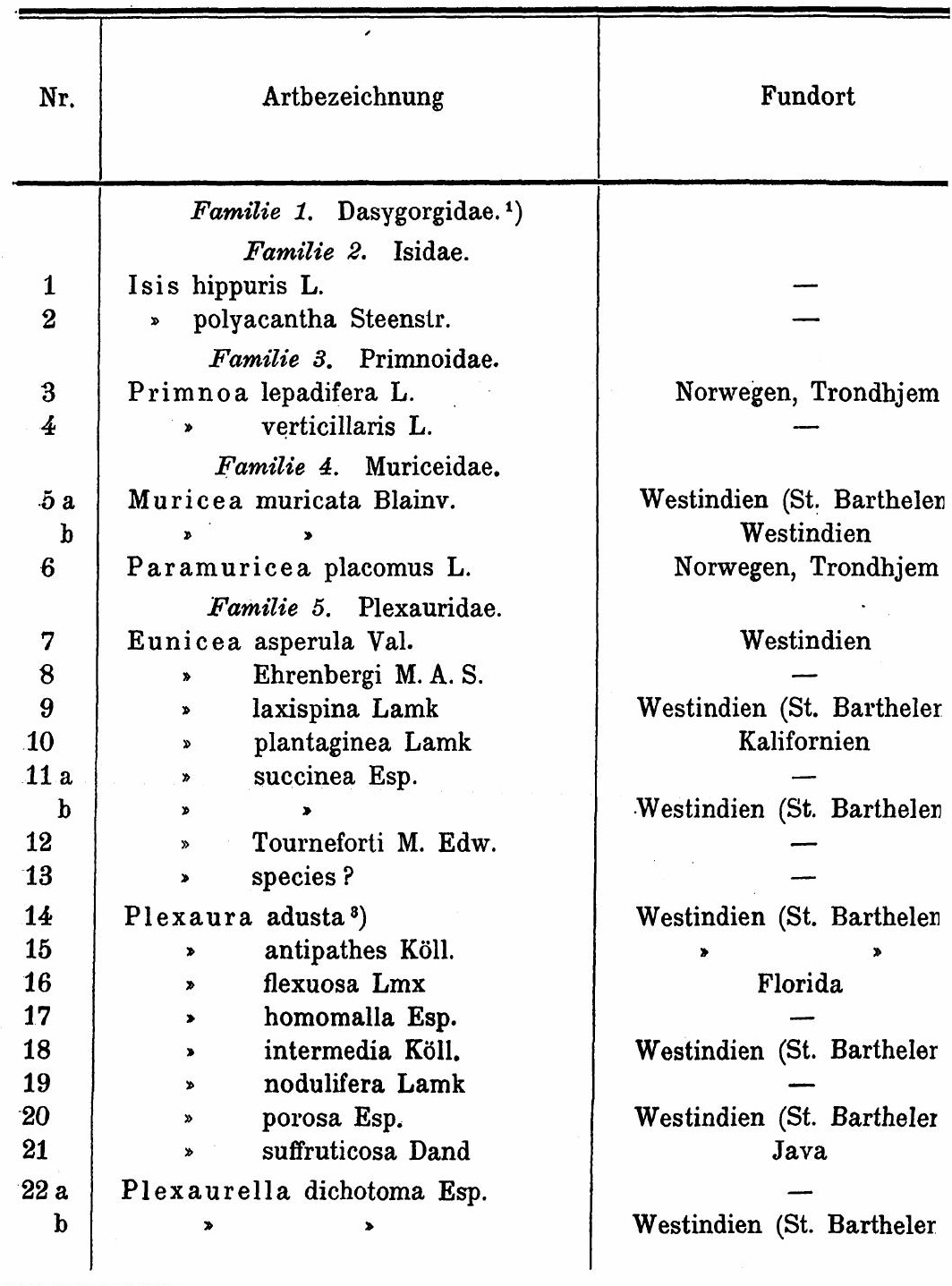

1) Die Familien kommen in der Reihenfolge vor, wie sie von Studer [R., Aus praktischen Gründen sind auch die Schwefelwerte aufgeführt worden, $c$ Alle Zahlen beziehen sich auf Trockensubstanz (wo Aschengehalt sich ang

2) Material von dieser Familie hat mir nicht zu Gebote gestanden.

s) Sammlungen des Reichsmuseums, Stockholm. 
Über die organische Gerüstsubstanz des Anthozoënskeletts. I.

lle.. $\left.{ }^{1}\right)$

\begin{tabular}{|c|c|c|c|c|c|c|c|}
\hline \multirow{2}{*}{$\begin{array}{l}\text { zahhl } \\
\text { ler } \\
\text { emn- } \\
\text { aree }\end{array}$} & \multirow{2}{*}{$\begin{array}{c}\text { Total- } \\
\text { gewicht } \\
\text { des gerein } \\
\text { Materials } \\
\text { g }\end{array}$} & \multirow{2}{*}{$\begin{array}{l}\text { Quali- } \\
\text { tative } \\
\text { Prüfung } \\
\text { auf } \mathrm{Br}\end{array}$} & \multicolumn{3}{|c|}{$\begin{array}{c}\text { Haloidgehalt } \\
\%\end{array}$} & \multirow{2}{*}{$\begin{array}{c}\text { S-Gehalt } \\
\%\end{array}$} & \multirow{2}{*}{$\begin{array}{c}\text { Asche- } \\
\text { gehalt } \\
\%\end{array}$} \\
\hline & & & $\mathbf{J}$ & $\mathrm{Br}$ & $\mathrm{Cl}$ & & \\
\hline 一 & - & - & - & - & - & - & - \\
\hline - & - & positiv & 2,03 & - & - & - & 0,22 \\
\hline$\longrightarrow$ & - & > & 1,58 & 0,74 & 0,10 & - & 0,13 \\
\hline 1 & 76,9 & . & 0,12 & 3,76 & 0,08 & 1,28 & 0,12 \\
\hline - & - & > & 0,05 & 2,94 & 0,07 & - & Spur \\
\hline 1 & 1,9 & $\triangleright$ & 0,30 & - & - & - & 0,17 \\
\hline 1 & 0,9 & » & 1,06 & - & - & - & - \\
\hline 1 & 15,3 & $>$ & 0,25 & 1,18 & 0,11 & 1,55 & 0,07 \\
\hline 1 & 6,8 & , & 1,25 & 3,70 & 0,12 & 1,22 & Spur \\
\hline 1 & 13,5 & » & 1,72 & 4,20 & 0,31 & 1,07 & 0,16 \\
\hline 1 & 6,8 & $\triangleright$ & 1,45 & 2,86 & 0,29 & 1,10 & 0,05 \\
\hline 1 & 2,9 & » & 1,03 & - & - & - & 0,06 \\
\hline 2 & 5,5 & $>$ & 1,06 & 2,89 & 0,21 & 1,19 & 0,04 \\
\hline 1 & 5,9 & > & 1,04 & 3,02 & 0,10 & 1,05 & Spur \\
\hline 1 & 2,1 & 》 & 1,50 & - & - & - & Spur \\
\hline- & - & $>$ & - & - & - & - & - \\
\hline 1 & 5,3 & , & 1,55 & - & - & - & 0,12 \\
\hline 1 & 16,2 & > & 0,88 & 3,50 & 0,14 & 1,16 & 0,29 \\
\hline 1 & 7,4 & > & 1,23 & 3,47 & 0,28 & 1,12 & 0,16 \\
\hline 1 & 16,8 & > & 0,84 & 3,65 & 0,12 & 1,24 & 0,08 \\
\hline 1 & 38,0 & • & 1,34 & 3,05 & 0,17 & 1,29 & 0,11 \\
\hline 1 & 6,1 & " & 0,74 & 3,02 & 0,38 & 1,34 & 0,06 \\
\hline 1 & 2,7 & , & 1,03 & - & - & - & 0,13 \\
\hline 1 & 1,2 & $\triangleright$ & 0,58 & - & - & - & Spur \\
\hline 1 & 8,8 & $\triangleright$ & 0,12 & 1,07 & 0,06 & - & 0,15 \\
\hline 1 & 22,9 & $\triangleright$ & 0,11 & 0,96 & 0,09 & - & 0,14 \\
\hline
\end{tabular}

wandat wird, die Gattungen und Arten im allgemeinen in alphabetischer Reihenfolge. veiterre Behandlung derselben erst in einer späteren Abteilung geschieht. t, auf asehenfreie Trockensubstanz). 


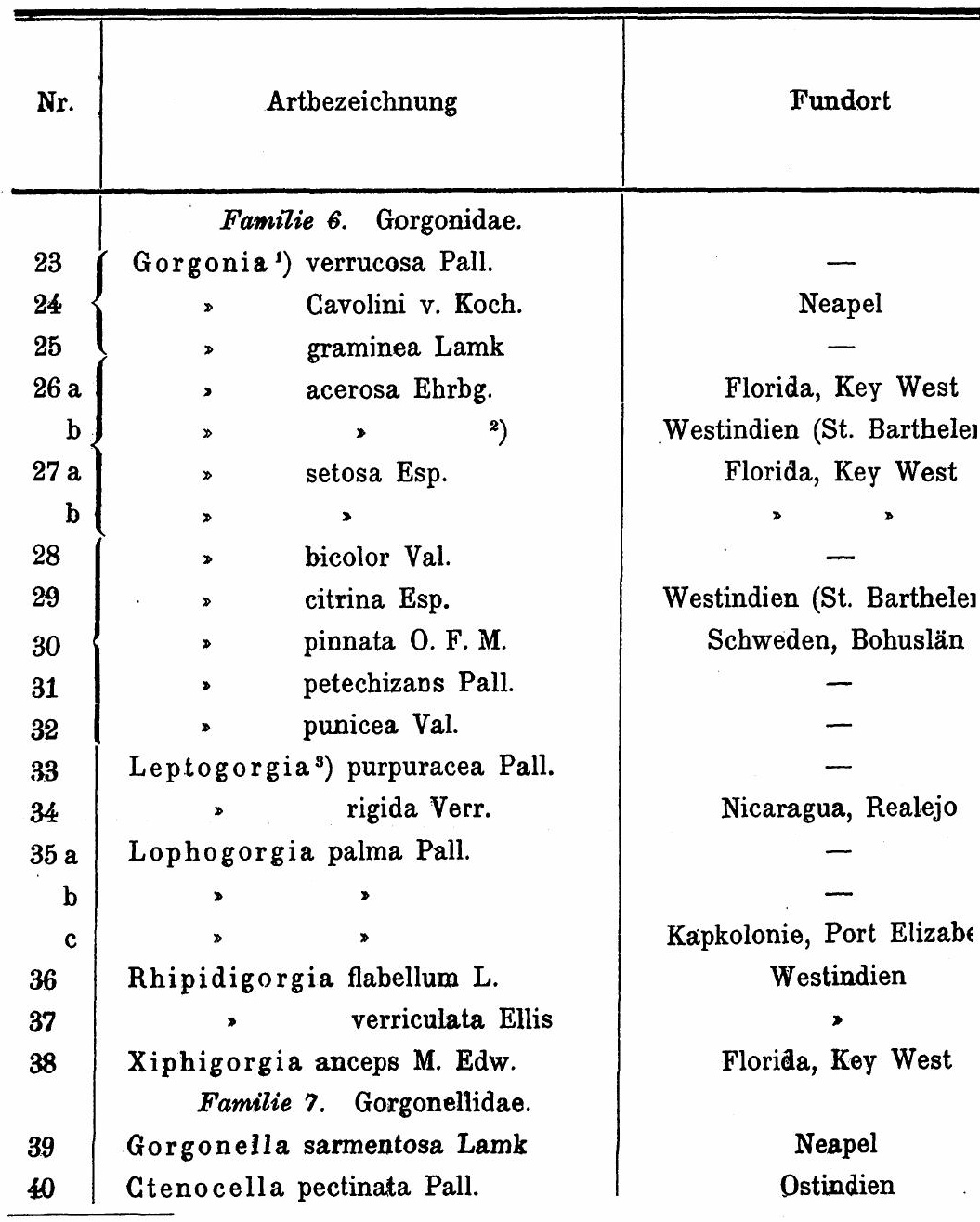

1) In älterer Zeit wurde die große Mehrzahl von Gorgonacéen zu einer homogene Gruppen ausgeschieden worden : Primnoa, Eunicea, Plexaura u. a. r demnach noch sehr heterogen. Doch können darin zwei kleinere Gruppen vonei graminea und verrucosa - siehe v. Koch [K., S. 58] - teils Gorgonia acero

Was übrig bleibt - eine exquisit heterogene Sammlung - habe ic

*) Das fragliche Material ist unter der Benennung Pterogorgia pinnata Milne Edwards [P., S. 168], auf Gorgonia acerosa Ehrbg. zu beziehen.

s) Die beiden hier aufgenommenen Arten sind, als im allgemeinen I 
Hlle.

(Fortsetzung.)

\begin{tabular}{|c|c|c|c|c|c|c|c|}
\hline \multirow{2}{*}{$\begin{array}{l}\text { azalahl } \\
\text { der:r } \\
\text { xemm- } \\
\text { larere }\end{array}$} & \multirow{2}{*}{$\begin{array}{c}\text { Total- } \\
\text { gewicht } \\
\text { des gerein. } \\
\text { Materials } \\
\text { g }\end{array}$} & \multirow{2}{*}{$\begin{array}{l}\text { Quali- } \\
\text { tative } \\
\text { Prüfung } \\
\text { auf } \mathrm{Br}\end{array}$} & \multicolumn{3}{|c|}{$\begin{array}{c}\text { Haloidgehalt } \\
\%\end{array}$} & \multirow{2}{*}{$\begin{array}{c}\text { S-Gehalt } \\
\%\end{array}$} & \multirow{2}{*}{$\begin{array}{c}\text { Asche- } \\
\text { gehalt } \\
\%\end{array}$} \\
\hline & & & $\mathrm{J}$ & $\mathrm{Br}$ & $\mathrm{Cl}$ & & \\
\hline 3 & 19,7 & positiv & 6,92 & 1,62 & 0,17 & 1,00 & 0,18 \\
\hline - & - & $\triangleright$ & 5,49 & 1,98 & 0,16 & 0,94 & 0,04 \\
\hline 1 & 11,5 & » & 5,58 & 1,31 & 0,12 & 1,06 & 0,08 \\
\hline 1 & 33,5 & , & 0,90 & 0,66 & 0,04 & 0,93 & 0,43 \\
\hline 1 & 5,3 & $\triangleright$ & 1,53 & 0,82 & 0,04 & 1,04 & - \\
\hline 2 & 130,0 & , & 0,77 & - & - & 0,89 & 0,14 \\
\hline 2 & 104,7 & , & 0,70 & 0,59 & 0,18 & 0,94 & 0,02 \\
\hline 1 & 4,6 & , & 0,23 & 0,88 & 0,05 & - & 2,31 \\
\hline 2 & 2,2 & , & 0,79 & - & - & - & 0,07 \\
\hline- & - & , & 0,02 & - & - & - & - \\
\hline 1 & 1,1 & , & 0,61 & - & - & - & - \\
\hline 2 & 0,7 & $\triangleright$ & 0,20 & - & - & - & - \\
\hline 1 & $.2,6$ & $\triangleright$ & 0,28 & 2,61 & 0,11 & - & - \\
\hline 1 & 4,0 & , & 0,24 & 0,99 & 0,05 & - & 0,86 \\
\hline - & - & , & 0,07 & 2,59 & 0,11 & - & 2,76 \\
\hline 1 & 11,6 & , & 0.08 & 2,30 & 0,09 & 1,23 & 2,44 \\
\hline 2 & 0,8 & , & 0,03 & - & - & - & - \\
\hline 2 & 43,6 & , & 0,45 & 0,37 & 0,05 & 0,81 & 0,50 \\
\hline 1 & 6,3 & , & 0,62 & 0,75 & 0,04 & 0,90 & Spur \\
\hline 1 & 8,7 & , & 0,96 & 0,23 & 0,17 & 1,17 & 0,13 \\
\hline 1 & 9,9 & , & 0,12 & 1,98 & 0,04 & 1,39 & 3,19 \\
\hline 1 & 9,5 & , & 2,21 & 0,66 & 0,16 & 1,18 & 0,14 \\
\hline
\end{tabular}

Ben 'Gattung, Gorgonia, vereinigt. Später sind daraus mehr differenzierte und enmaaterial, das hier immer noch unter der Gorgonia-Gattung aufgeführt wird, ist r nałhestehenden Arten unterschieden werden, nämlich teils Gorgonia Cavolini, ssa.

einee dritte Gruppe aufgenommen.

altenn worden und ist daher vermutlich, nach der Synonymverzeichnung bei $r$ voøn einander verschieden, nicht in näherer Weise verwandt. 
nahme, mehr oder weniger unzuverlässig, in gewissen Fällen in sehr hohem Grade irreführend. Die anfangs von Drechsel angewandte und später von Mendel und (teilweise) von Cook befolgte Analysenmethode (Erhitzen des gesamten Haloidsilberniederschlages im Cl-Strom) hat nämlich zur unerläßlichen Voraussetzung, daß $\mathrm{Br}$ in dem Analysenmaterial nicht vorhanden ist. Im entgegengesetzten Falle muß das anwesende $\mathrm{Br}$, je nach seiner Menge, die für $\mathrm{J}$ berechnete Zahl erhöhen. Bei relativ geringer J- und großer Br-Menge muß sich die gefundene Zahl zu einer wahren Karikatur gestalten, verglichen mit den faktischen Verhältnissen. Als typisches Beispiel sei folgendes angeführt.

Durch Analyse nach Drechsels Verfahren bestimmte Cook den J-Gehalt in Plexaura flexuosa auf 5,0\%. ${ }^{1}$ ) Nimmt man nun an, daß das Analysenmaterial den Br-Gehalt, 3,5\%, gehabt hat, wie er von mir bei derselben Art gefunden worden ist - welcher Br-Gehalt bei der Analysenberechnung einen J-Gehalt von $2,7 \%$ simuliert -, so hätte der wirkliche JGehalt nur 2,3\% betragen.

Cook hat sich auch einer auf einem anderen Prinzip basierenden J-Bestimmungsmethode, der An d re w schen, bedient. $\mathrm{Da}$ indessen diese Methode, nach von Cook ausgeführten vergleichenden Bestimmungen zu urteilen, zu Ergebnissen führt, die nahezu mit den an demselben Material nach Drechsels Methode erhaltenen zusammenfallen - sogar betreffs der so sehr Br-reichen Plexaura flexuosa -, so muß ich daraus den Schluß ziehen, daß - wenigstens bei der Weise, wie Cook sie angewandt ${ }^{2}$ ) - auch bei dieser Methode anwesendes $\mathrm{Br}$ (Bromid) in demselben Grade und in derselben Richtung sich geltend macht.

1) [B. S. 98.] Die Umrechnung für aschenfreie Substanz stammt von mir her (Originalzahlen: $4,63 \% \mathrm{~J} ; 7,76 \%$ Asche).

$\left.{ }^{2}\right)$ Cook sagt, er habe a modification of the quantitative method of L. W. Andrews angewandt (ohne anzugeben, worin die Modifikation bestanden hat).

Beiläufig sei bemerkt, daß bei Anwendung der Andrewschen Methode auf angesäuerte Lösung von Alkalischmelze eine vom Haloidgehalt unabhängige Fehlerquelle vorhanden ist, die auf $\mathrm{zu}$ hohe Titrierungsresultate hintendiert; ich denke an aus dem Proteinschwefel ent- 
Auf Grund der angeführten Umstände müssen im folgenden die älteren quantitativen J-Bestimmungen fast ganz unberücksichtigt bleiben.

Da nach dem oben Angeführten der Gehalt an J in hohem Grade verschieden groß ist bei verschiedenen Arten, so ist es natürlich wichtig, wenn es sich um Versuche handelt, das organische J-haltige Spaltungsprodukt, Jodgorgosäure, darzustellen, dafür zu sorgen, daß man sein Ausgangsmaterial von einer geeigneten (möglichst J-reichen) Art erhält. Es ist daher als ein glücklicher Zufall anzusehen, daß gerade Gorgonia Cavolini (als in der Umgegend von Neapel reichlicher vorkommend) Drechsel und später Henze zu Gebote gestanden hat. Mit der einen oder anderen der großen Mehrzahl von Gorgonaceen als Material wäre sicherlich die Arbeit lange genug fruchtlos geblieben, wie es auch Mendel geschah (mit Gorgonia flabellum als Material). ${ }^{1}$ )

B) Über das Vorkommen von Brom.

Während der Br-Gehalt in dem Material (Gorgonia setosa), wo er von mir zuerst beobachtet wurde, relativ niedrig war $(0,59 \%)$, erhielt ich einen Begriff von der großen Bedeutung des $\mathrm{Br}$ als Konstituent im Gorgonaceenskelett schon bei der Analyse, die darauf zunächst folgte, indem der Zufall es fügte, daß das Material dabei aus Primnoa lepadifera (mit nahezu $4 \% \mathrm{Br}$ ) bestand. In entschiedenem Gegensatz zu dem, was bisher angegeben worden, hat jede der nunmehr analysierten 40 Arten (Dubletten einberechnet: $47 \mathrm{Spe}-$ cimina) sich als $\mathrm{Br}$ in organischer Bindung enthaltend erwiesen. Die beobachtete Menge davon hat sich zwischen 0,23 und $4,20 \%$ bewegt. Es ist zu bemerken, daß für den Nachweis des Broms keine reichlichere Materialquantität erforderlich gewesen ist; die für die qualitative Prüfung angewandte

standenes Alkalisulfid (resp. $\mathrm{H}_{2} \mathrm{~S}$ nach der Ansäuerung). Während des Schmelzens darf nämlich nicht zur vollständigen Oxydierung des $S$ erforderliches Nitrat zugesetzt werden (indem sich bildendes Nitrit in solchem Falle $J$ freimachen und $\mathrm{zu}$ unberechenbaren Fehlern $-\mathrm{zu}$ nie=drigen Titrierungsresultaten - Anlaß geben würde).

1) [0. S. 245]. 
Materialmenge, in der Regel $1 \mathrm{~g}$ betragend, ist in keinem Fall größer gewesen, in mehreren Fällen dagegen kleiner ( $3 / 4$ oder $1 / 2$ g). Unter solchen Umständen erhebt sich natürlich die Frage, weshalb die früheren Forscher, die offenbar auf die Br-Prüfung ihres Materials alle Sorgfalt verwendet haben, sogar bei der Bearbeitung einer so großen Materialquantität wie $30 \mathrm{~g}$ ! (Mendel), $\left.{ }^{1}\right)$ absolut negative Resultate erhalten haben. Hierüber wage ich mich nicht mit Bestimmtheit zu äußern, doch möchte ich auf Grund eigener Erfahrungen - es für das Wahrscheinlichste halten, daß die Erklärung sehr einfacher Art ist. Im Laufe der Arbeit habe ich - bei der qualitativen Br-Prüfung, die ich an der (durch Ausschütteln mit $\mathrm{CS}_{2}$ vollständig von $\mathrm{J}$ befreiten) $\mathrm{N}_{2} \mathrm{O}_{3}$ - haltigen ${ }^{2}$ ) Lösung anstellte - jedesmal beobachtet, daß eine gewisse, je nach der Größe des vorhandenen $\mathrm{N}_{2} \mathrm{O}_{3}$-Gehalts wechselnde Menge $\mathrm{Cl}$-Wasser zugesetzt werden kann, ohne daß dadurch $\mathrm{Br}$ freigemacht wird. Erst wenn das vorhandene $\mathrm{N}_{2} \mathrm{O}_{3}$ vollständig zersetzt worden ist (nach dem Schema: $\mathrm{N}_{2} \mathrm{O}_{3}+4 \mathrm{Cl}+2 \mathrm{H}_{2} \mathrm{O}=\mathrm{N}_{2} \mathrm{O}_{5}+4 \mathrm{HCl}$ ), vermag eine weitere, vorsichtig zugemessene ${ }^{3}$ ) Menge Cl-Wasser $\mathrm{Br}$ aus anwesendem Bromid freizumachen. Beobachtet man diesen Umstand nicht, und ist überdies während der Schmelzung an Salpeter nicht gespart worden, so können kopiöse Mengen Cl-Wasser mit vollkommen negativem Resultat in bezug auf Br-Reaktion zugesetzt werden, trotzdem die Lösung tatsächlich Bromide enthält.

Ein Detail, das bei künftiger Arbeit bei der Darstellung der Jodgorgosäure Aufmerksamkeit verlangt, ist dies, daß das Endprodukt in bezug auf eventuellen Gehalt an $\mathrm{Br}$ zu prüfen ist. Eine Verunreinigung durch eine der Jodgorgosäure (= Dijod-

1) [0. S. 245].

2) Teils von in der Schmelze (aus $\mathrm{NaNO}_{8}$ ) entstandenem Nitrit, teils von zur Freimachung des $\mathrm{J}$ in etwas Überschuß zugesetzer $\mathrm{N}_{2} \mathrm{O}_{8}$ Schwefelsäure herrührend.

3) Andererseits kann, wie bekannt, der Br-Nachweis durch allzu reichlichen Cl-Wasserzusatz gefährdet werden, indem sich dabei die Verbindung $\mathrm{BrCl}$ bildet, deren Lösung in $\mathrm{CS}_{2}$ eine wenig markierte, grüngelbe Farbe besitzt. 
tyrosin) analoge Bromgorgosäure (Dibromtyrosin?), die während der Hydrolyse entstanden wäre, scheint nämlich durchaus nicht undenkbar. Andererseits muß man - bei einem zielbewußten Versuch, ein derartiges, eventuell auftretendes Br-haltiges, hydrolytisches Spaltungsprodukt aus dem Gorgonaceenskelett zu isolieren - eine möglichst Br-reiche (und gleichzeitig möglichst J-arme) Art zu wählen, z. B. Primnoa lepadifera. ${ }^{1}$ )

r) Über das Vorkommen von Chlor.

Der Gehalt an $\mathrm{Cl}$ hat sich im Gegensatz zu dem, was frühere Untersuchungen an die Hand zu geben scheinen, als äußerst unbedeutend, ein bis ein paar Zehntel Prozent, erwiesen. Die Größe der von Drechsel und Mendel angegebenen ClWerte (1-2 bis zu 3\%) ist offenbar durch das Zusammenwirken zweier, nunmehr offen zutage liegenden Fehlerquellen entstanden:

teils des eigenen Gehalts des nicht vorbehandelten (extrahierten)Untersuchungsmaterials an anorganischem Chlor(Chlorid),

teils der zur Cl- (und J-)Bestimmung angewandten Methode, die - bei faktischer Gegenwart von (nicht beachtetem) $\mathrm{Br}$ - zu hohe Resultate für $\mathrm{Cl}$ (wie auch für $\mathrm{J}$ ) geben muß.

ठ) Über das Haloidvorkommen im übrigen.

Beim Anblick der bunten Zahlenwerte der Analysentabelle möchte man vielleicht geneigt sein, einen größeren oder geringeren Haloidgehalt als ganz zufällig entstanden anzusehen. Ein genaueres Studium läßt jedoch etwas anderes erkennen. Ziemlich deutlich tritt uns das Gesetz entgegen, daß der Haloidgehalt a) bei verschiedenen Individuen derselben Art und b) bei morphologisch sehr nahestehenden Arten, wenn auch nicht ohne Schwankungen, so doch im großen und ganzen als von derselben Größenordnung bezeichnet werden kann, daß, mit anderen Worten, dieser Faktor (es gilt dies sowohl für $\mathrm{J}$ als auch für $\mathrm{Br}$, jedes für sich) in seiner Weise einen Artcharakter darstellt, d. h. überhaupt ein Zeichen morphologischer Ver-

1) Um hierüber, wenn möglich, Klarheit zu gewinnen, habe ich gegenwärtig ein relativ reichliches Material (mehrere $100 \mathrm{~g}$ ) von der letztgenannten Art unter Vorbehandlung. 
wand tschaft ist. $\left.{ }^{1}\right)$ Wenn auch, auf Grund des oben Angeführten (siehe S. 50), den aus früheren Untersuchungen hervorgegangenen J-Werten im allgemeinen keine Gültigkeit beigemessen werden kann, so dürften doch Cooks ${ }^{2}$ ) Zahlen für 3 verschiedene Specimina von Plexaura flexuosa - 5,6, 5,0, resp. 5,7\% - da sie auf einer und derselben Analysenmethode (Andrews) beruhen und überdies sämtliche für aschenfreie Trockensubstanz umgerechnet sind, bei Vergleich unter einander zur Beleuchtung der vorliegenden Frage einen gewissen Wert haben.

Ob die Übereinstimmung in dieser Hinsicht ihrerseits ihren Grund in einer Übereinstimmung der äußeren Lebensbedingungen haben kann(Größe des Wasserdrucks, Lichtintensität, Beschaffenheit des Planktons usw. an den Lokalitäten, wo die Art vorzugsweise vorkommt), ist mir gegenwärtig unbekannt. So viel scheint jedoch bereits aus den vorliegenden Fakta hervorzugehen, daß von derartigen denkbaren Momenten wenigstens eines als nicht in höherem Grade oder wenigstens nicht allein bestimmend eliminiert werden kann, nämlich das Klima bzw. der Breitengrad. Man vergleiche z. B. einerseits Nr. 3 und 4, die eine aus dem Trondhjemsfjord in Norwegen $\left(63^{\circ} \mathrm{n}\right.$. Br.), die andere aus dem Mittelmeer, und doch beide sehr nahe Übereinstimmung hinsichtlich des Haloidgehalts aufweisend, andererseits $\mathrm{Nr} .16$ (Br: 3,47\%) mit Nr. 38 (Br: 0,23\%) - beide von Florida, oder Nr. $11 \mathrm{~b}(\mathrm{~J}: 1,04 ; \mathrm{Br}: 3,02 \%)$ mit Nr. $22 \mathrm{~b}(\mathrm{~J}: 0,11 ; \mathrm{Br}$ : $0,96 \%)$ - beide von St. Barthelemy oder Nr. 24 (J: 5,49\%) mit Nr. 39 (J: 0,12\%) - beide von Neapel usw. Es schien dieses Verhältnis mir der Erwähnung wert, weil Hunde shag en,$^{3}$ ) auf seine Untersuchungen über Spongien sich stützend, den generell gehaltenen Satz ausspricht: «Das heiße Klima scheint

1) Zur Verifizierung des Gesagten betrachte man z. B. teils Nr. 5, 11, 22, 26, 27 und 35, welche alle in Dubletten vorliegen, teils die von den übrigen Gruppen wohldifferenzierten Gattungen Primnoa (Nr. 3-4), Eunicea + Plexaura (Nr. 7-21) und Rhipidigorgia (Nr. 36-37), wie auch innerhalb der Gattung Gorgonia die einander sehr nahestehenden Artgruppen Nr. 23-25 bezw. 26-27.

2) [B. S. 98].

${ }^{8}$ ) [J. S. 474]. 
für die Anreicherung des Jods in der organischen Substanz der Meeresbewohner eine wesentliche Bedingung zu sein».

Mit großer Wahrscheinlichkeit ist wohl, der soeben gegebenen Zusammenstellung nach zu urteilen, auch ein anderes Moment, das a priori als entscheidenden Einfluß ausübend gedacht werden könnte, der Hauptsache nach als eliminiert zu betrachten, nämlich der Unterschied im Gehalt des Meereswassers an Haloiden an den Orten für das Vorkommen der verschiedenen Arten. Man vergleiche die Beispiele, die oben aus so begrenzten Lokalitäten wie St. Barthelemy und Neapel angeführt worden sind.

Nach einer Bemerkung von Harnack ${ }^{1}$ ) scheint für die von ihm untersuchten Spongien zu gelten, daß der Jodgehalt mit dem Alter des Skeletts zunimmt. Eine entsprechende, auf Untersuchung gegründete Angabe betreffs der Gorgonaceen (= Hornkorallen) oder anderer Korallen liegt meines Wissens in der Literatur nicht vor. Die folgende Bemerkung von Cohnheim: ${ }^{2}$ ) «Das Gorgonin — - enthält - - fast $8 \%{ }^{3}$ ) organisch gebundenes Jod; ältere, festere, Gerüstteile vielleicht noch viel mehr * und * Junge _ _ - Korallen enthalten - - erheblich weniger Jod» dürfte demnach nur auf spekulativem Wege entstanden sein, unter dem Einfluß der nur für gewisse Spongien geltenden Harnackschen Erfahrungen. Wie es in Wirklichkeit hiermit sich verhält, habe ich (auch betreffs des Br-Gehalts) näher zu erforschen gesucht. Zunächst können zur Beleuchtung der Frage aus der Analysentabelle die, freilich wenigen, Fälle herangezogen werden, wo Doppelexemplare von derselben Art, die hinsichtlich der Größe in höherem Grade von einander

1) [G. S. 413-414]. «Der Jodgehalt in der organischen Rohsubstanz des Badeschwammes ist, namentlich in älteren, größeren Exemplaren, kein unerheblicher s. ¿Dagegen erhielt ich aus ganz kleinen Schwämmen nur etwa die Hälfte dieser Menge; es scheint demnach der Jodgehalt mit dem Alter zu steigen - $\rightarrow$.

2) [C. S. 120-121].

8) Daß diese Angabe über die Größe des J-Gehalts durchaus nicht allgemeingültig ist, ergibt sich mit aller Deutlichkeit sowohl aus den früheren Untersuchungen (von Mendel und Cook) als aus den hier vorgelegten. 
verschieden waren, zur Untersuchung gekommen sind [siehe Nr. 22b) und a), 26a) und b), 35b) und c)]. Aber auch auf anderem Wege habe ich versucht, Anhaltspunkte zur Beurteilung der Frage zu gewinnen. Von verschiedenen Arten sind dieselben Exemplare von Antang an auf eine solche Weise zerstückelt worden, daß jüngere und ältere Teile je für sich genommen wurden, worauf die verschiedenen Partien gesondert vorbehandelt und analysiert wurden. Die Resultate der Versuche werden in der Tabelle auf Seite 57 mitgeteilt.

Aus diesen beiden Zusammenstellungen geht hervor, daß, was die Gorgonaceen betrifft, ein augenfälliger Unterschied im Haloidgehalt zwischen jüngeren und älteren Skelettpartien derselben Art nicht vorhanden ist. Höchstens läßt sich von der Andeutung eines Unterschiedes sprechen, der in derselben Richtung wie der bei gewissen Spongien sehr markant hervortretende geht. Hauptsächlich, kann man also sagen, ist Cohnheims präsumtive Äußerung betreffs des J-Gehalts nicht bestätigtworden.

Bis auf weiteres darf man sich wohl als nicht unwahrscheinlich denken, daß die organischen Gerüstsubstanzen von verschiedenen Arten höhere oder niedere Haloidsubstitutionsderivate eines der Hauptsache nach gemeinsamen, zugrunde liegenden Proteinstoffs (Gorgonin) darstellen. Und in diesem spezieilen Sinne würde sogar Krukenbergs sonst - wie wir nunmehr wissen - unverständliche Bemerkung, ${ }^{1}$ ) daß das *Cornein v von allen Arten dasselbe wäre, einen gewissen Grad von Berechtigung behalten.

1) [L. S. 15; M. S. 1844]. Die Bemerkung gründet sich wesentlich auf Elementaranalysen. Schwer zu erklären ist allerdings, daß diese bezüglich des Corneins von 1. Rhipidigorgia flabellum und 2. Gorgonia verrucosa so vollständig übereinstimmen:

\begin{tabular}{c|c|c|c|c}
\hline Nr. & C & H & N & O \\
\hline 1 & 48,94 & 5,80 & 17,06 & 28,19 \\
2 & 49,02 & 5,81 & 16,76 & 28,40
\end{tabular}

ob ohl - wie nunmehr bekannt ist - Nr. 1 einen Haloidgehalt von $8-9 \%$, Nr. 2 dagegen nur einen solchen von ungefähr $1 \%$ besitzt! 


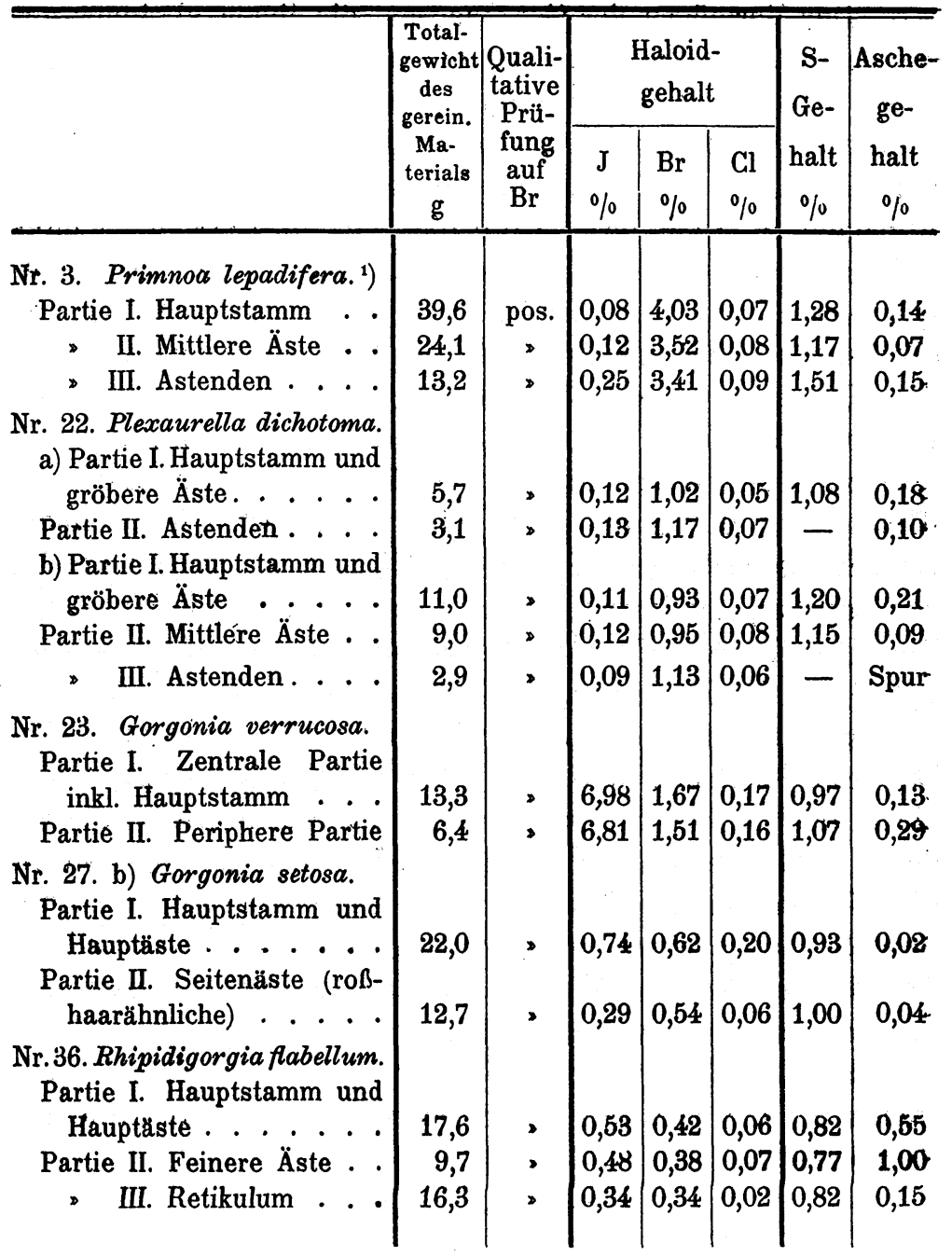

1) Die in dieser Tabelle aufgeführten Analysenwerte (für aschefreie Trockensubstanz geltend) sind die primären. Die in der Haupttabelle (S. 46-49) für dieselben Arten angegebenen Durchschnittszahlen sind aus diesen ersteren berechnet, unter gebührender Berücksichtigung. des Gewichts der verschiedenen Materialpartien. (Um eine besondere Tabelle für den S-Gehalt im nächsten Kapitel zu ersparen, sind die $\mathbf{S}$ Werte bereits hier angeführt worden). 
Das Vermögen der Gorgonaceen, die ihnen als Ionen nur spurenweise zur Verfügung stehenden Haloide des Meerwassers, $\mathrm{J}$ und $\mathrm{Br}$, zu entionisieren und aufzunehmen, um sie in konzentrierter Form in ihrem Skelett (nicht im Coenenchym) zu deponieren, ist ja höchst bemerkenswert. Drechsel neigt der Annahme zu, «daß diese Tiere das Jod zur Herstellung ihres Achsenskeletts notwendig brauchen». Zwar finden sich ziemlich wesentliche Verschiedenheiten in physikalischer Hinsicht (Farbennüance; Texturdichte, Härte, Elastizität usw.) zwischen dem Gorgonin von verschiedenen Arten oder von älteren und jüngeren Partien derselben Art, irgend einen Zusammenhang aber zwischen diesen Faktoren und dem J-, dem Br- bezw. dem totalen Haloidgehalt habe ich nicht feststellen können. Eine Stütze für Drechsels Ansicht bildet dieser Umstand nicht, sondern eher, soweit ich sehen kann, das Gegenteil. Die Frage nach der eigentlichen Bedeutung dieses eminenten Haloidaufnahmevermögens der Gorgonaceen, d. h. ob es eine notwendige Voraussetzung für ihre normale Entwicklung und Lebensfunktionen ist, resp. überhaupt dabei eine wesentliche Rolle spielt oder nicht, dürfte definitiv kaum anders zu beantworten sein als durch Kulturversuche mit jungen Gorgonaceenexemplaren in artifiziellem, J- resp. Br-freiem Meerwasser. Eine solche experimentelle Untersuchung müßte meines Erachtens eine dankbare Aufgabe sein, wert, daß sie an einer zoologischen Station unternommen würde, wenn es sich auch vielleicht zeigen sollte, daß ihrer Ausführung gewisse Schwierigkeiten sich entgegenstellen ( $u$. a. wegen des relativ langsamen Wachstums dieser Tierkolonien).

\section{B. Der Schwefelgehalt.}

1. Literaturübersicht.

Die Frage nach der Stellung der Gorgonaceen-Gerüstsubstanz (des Gorgonins) zur Keratingruppe ist eng mit der nach dem Schwefelgehalt dieser Substanz verknüpft. Die allerfrüheste Angabe (1855) betreffs einiger Verhältnisse des Gorgonins zu einfacheren chemischen Reagenzien rührt von Valenciennes ${ }^{1}$ )

1) [S. S. 11]. 
her. Dieser spricht es deutlich aus, daß er diese Substanz nicht für chemisch identisch mit der Gerüstsubstanz in den Hörnern, Nägeln oder Klauen ( «de la corne») der Säugetiere hält, sondern daß sie einen Stoff sui generis darstellt, obwohl er, auf Grund gewisser Übereinstimmungen zwischen ihnen, die Benennung Cornein vorschlägt. Von Schwefelgehalt wird nichts erwähnt. Valenciennes' Zeitgenosse Fremy ${ }^{1}$ ) dagegen hält die Gorgonaceensubstanz, die er als schwefelfrei angibt, für analog mit Conchiolin und bezeichnet sie auch mit demselben Namen. In Übereinstimmung mit Fremy gibt Krukenberg - der Valenciennes' Benennung Cornein akzeptiert und im übrigen keine bestimmte Ansicht über die Stellung des Gorgonins in systematischer Hinsicht ausgesprochen hat - die Substanz als schwefelfrei an. ${ }^{2}$ ) Hauptsächlich auf Grund des Studiums der hydrolytischen Spaltungsprodukte hat Drechsel ${ }^{3}$ ) das Gorgonin den Keratinsubstanzen der Wirbeltiere an die Seite gestellt. Von einem Versuch, durch eine Analysierung des Schwefelgehalts diese Diagnose zu stützen, wird jedoch nichts erwähnt. In letztgenannter Hinsicht hat Henze Drechsels Bemerkung zu komplettieren versucht und glaubt in dem erhaltenen Resultat - 2,32\% S - eine Bestätigung für sie erhalten zu haben. ${ }^{4}$ ) Schließlich hat Cook ${ }^{5}$ ) bei der S-Bestimmung an Material von mehreren verschiedenen Arten (7 Stück) Werte gefunden, die weit niedriger sind als die Henzes, im Durchschnitt 1,30\% ${ }^{6}$ ) (ohne im Anschluß daran eine bestimmte Ansicht über die Stellung des Gorgonins zu den Keratinen zu formulieren).

1) [E. S. 97].

2) : - - - da selbst größere Stücke, mit reinem Salpeter und reiner Soda verascht, nur wässerige Lösungen lieferten, in denen sich auf Zusatz von Chlorbaryum und Salzsäure kaum eine Trübung ausbildete» [L. S. 4].

s) [D. S. 95].

4) Wenn Henze [H. S. 62] sagt: «Es existieren darüber (= Schwefelgehalt) keine Angaben", so ist das ja nicht völlig exakt, indem sowohl Fremy als besonders Krukenberg das Gorgonin ausdrücklich als schwefelfrei angegeben haben.

s) [B. S. 97].

6) Niedrigster: 0,85 , höchster: $1,64 \%$. 


\section{Eigene Untersuchungen.}

In jedem untersuchten Fall ist die Substanz als schwefelhaltig befunden worden. ${ }^{1}$ ) Betreffs des von mir gefundenen S-Gehalts in Material von einer größeren Anzahl verschiedener Arten sei auf die Tabelle auf S. 46-49 verwiesen. Die Durchschnittszahl für 23 in der genannten Hinsicht untersuchte Arten beträgt $1,14 \% 0^{2}$ ) Dieser Wert stimmt ziemlich gut mit dem entsprechenden Cooks überein. Indessen ist zu beachten, daß auch diese beiden Durchschnittswerte wenigstens etwas zu hoch sein müssen. Cooks Analysen sind nämlich an durch Extraktion nicht vorbehandeltem Material ausgeführt, meine dagegen an Material, das zwar eine gründliche Vorbehandlung mit verdünnter Essigsäure erfahren hat, womit es jedoch nicht in jedem Fall gelungen 1st, inkrustiertes $\mathrm{CaSO}_{4}$ ganz vollständig zu entfernen (wie ich durch Kontrollversuche an dem Analysenmaterial ${ }^{3}$ ) mit verdünnter $\mathrm{HCl}$ erfahren habe). Im Durchschnitt dürfte daher der S-Gehalt des Gorgonins nicht auf höher als ca. $1 \% \mathrm{zu}$ veranschlagen sein.

Unter soIchen Verhältnissen steht Henzes Angabe über relativ hohen S-Gehalt ganz isoliert da. Es kann meines Erachtens kein Zweifel darüber obwalten, daß dabei irgend ein ungünstiger Umstand sich geltend gemacht hat, vielleicht hoher Sulfatgehalt in dem, der Beschreibung nach zu urteilen, nicht dekalzinierten Material oder ein Anlaß anderer Art (das für die Analyse abgewogene Material betrug nur $1 / 2$ g). An einem begründeten Anlaß, in Übereinstimmung mit Drechsel und Henze, die Gerüstsubstanz der Gorgonaceen der Gruppe der

1) Über die Ursache zu Fremys negativen Resultaten, die aus einer Zeit stammen, wo die Analysenmethoden wenig entwickelt waren, wage ich keine Ansicht auszusprechen. Krukenbergs völlig negative Resultate (siehe S. 59) dürften wahrscheinlich in unvorsichtigem Zusatz von $\mathrm{HCl}$ bei der Ansäuerung der Lösung der alkalischen Schmelze oder in ungenügend langer Observationszeit ihren Grund haben, indem, wie bekannt, $\mathrm{BaSO}_{4}$ aus $\mathrm{zu}$ stark $\mathrm{HCl}$-saurer Lösung unvollständig oder bei anwesender reichlicher Chloridmenge nur zögernd ausfällt.

2) Niedrigster Wert: 0,81, höchster: 1,55\%.

3) Dem mit mehr augenfälligem Aschengehalt. 
Keratine zuzuzählen, scheint es mir daher zu fehlen, wenn auch gewisse Übereinstimmungen, besonders in physikalischer Hinsicht, vorhanden sind.

\section{Zusammenfassung der Ergebnisse (die Gorgonaceen betreffend).}

1. In Übereinstimmung mit früheren Untersuchungen hat Jod sich als regelmäßig vorkommend erwiesen. In verschiedenen Fällen beobachteter Gehalt : 0,05-6,92\%. Am J-reichsten ist Gorgonia verrucosa nebst nahestehenden Arten (G. Cavolini und G. graminea).

Infolge des (unten zu erwähnenden) Br-Vorkommens sind alle bisherigen quantitativen J-Bestimmungen mehr oder weniger, bisweilen in allerhöchstem Grade irreführend.

2. Im Gegensatz zu den vollständig negativen Resultaten der früheren Untersucher hat sich auch Brom als ein konstanter Bestandteil erwiesen. Beobachteter Gehalt in verschiedenen Fällen: 0,23-4,20\%. Besonders Br-reich haben sich u. a. die Arten der Gattungen Primnoa, Eunicea und Plexaura gezeigt.

Es besteht Wahrscheinlichkeit für das Vorkommen einer der Jodgorgosäure analogen Bromgorgosäure.

3. Der Gehalt an Ghlor ist durchgehends ein unbedeutender gewesen (ein oder ein paar Zehntel Prozent). Die Ursache für die Unrichtigkeit älterer Angaben bezüglich hohen Cl-Gehalts (bis auf $3 \%$ ) ist nachgewiesen worden.

4. Alle die genannten Haloide sind organisch gebunden in der Gerüstsubstanz des Skeletts (Gorgonin) enthalten.

5. Der Totalgehalt an $\mathrm{J}+\mathrm{Br}$, resp. die Verteilung zwischen denselben, hat sich im großen und ganzen als eine Arteigenschaft, bezw. ein Zeichen für morphologische Verwandtschaft herausgestellt.

Diese Faktoren scheinen nicht von dem Klima (bezw. Breitengrad) der Standorte oder von dem eigenen Gehalt des vorhandenen Meerwassers an den gleichen Haloiden abzuhängen.

6. Ein augenfälliger Unterschied im Haloidgehalt zwischen älteren und jüngeren Skelettpartien von derselben Art ist nicht beobachtet worden (im Gegensatz zu dem, was, speziell für die Spongien, angegeben wird). 
7. Ein Zusammenhang zwischen den physikalischen Eigenschaften der Skelettgerüstsubstanz (Farbe, Dichte der Textur, Härte, Elastizität u. dgl.) und dem J-, dem Br-, bezw. dem totalen Haloidgehalt hat nicht bemerkt werden können.

8. Der Schwefelgehalt ist so niedrig - durchschnittlich ca. 1\% - daß die vorgeschlagene Zuweisung zur Gruppe der Keratine nicht in hinreichendem Grade motiviert erscheint.

Über die Untersuchung von Material aus den beiden übrigen Anthozoëngruppen, Pennatulacea und Alcyonacea, wird in einer späteren Mitteilung berichtet werden.

\section{Literaturverzeichnis.}

[A] Andrews, L., Titrations with potassium iodate. The journ. of the Americ. Chemic. Society, Bd. XXV (1903), S. 756-761.

[B] Cook, F., The chemical composition of some gorgonian corals. The americ. journ. of physiology, Bd. XII (1905), S. 95-98.

[C] Cohnheim, 0., Chemie der Eiweißkörper, 2. Aufl, Braunschweig 1904.

[D] Drechsel, E., Beiträge zur Chemie einiger Seetiere. II. Über das Achsenskelett von Gorgonia Cavolinii. III. Über das Jod im Gorgonỉn. Zeitschrift f. Biologie, Bd. XXXIII (1896), S. 90-107.

[E] Fremy, E., Recherches chimiques sur les os. Ann. de chimie et de physique, Bd. XLIII (1855), S. 47-107.

[F] Gorup-Besanez, E., Über die Einwirkung des Broms auf Tyrosin. Annal. d. Chemie u. Pharmacie, Bd. CXXV (1863), S. 281-291.

[G] Harnack, E., Über das Jodospongin, die jodhaltige, eiweißartige Substanz aus dem Badeschwamm. Diese Zeitschrift, Bd. XXIV (1898), S. 412-424.

[H] Henze, M., Zur Chemie des Gorgonins und der Jodgorgosăure. Diese Zeitschrift, Bd. XXXVIII (1903), S. 60-79.

[I] Hundeshagen, F., Über jodhaltige Spongien und Jodospongin. Zeitschrift f. angew. Chemie, Jahrg. 1895, S. 473-476.

[K] v. Koch, G., Die Gorgoniden des Golfes von Neapel und der angrenzenden Meeresabschnitte. Erster Teil einer Monographie der Anthozoa alcyonaria. Berlin 1887.

[L] Krukenberg, C. Fr. W., Zur Kenntnis der organischen Bestandteile der tierischen Gerüstsubstanzen (I. Mitteilung). Vergleich.physiol. Studien von Dr. G. Fr. W. Krukenberg, Abteil. 5, Heidelberg 1881, S. 1-37.

[M] - Über das Cornein. Berichte der Deutsch. chem. Gesellsch., Bd. XVII (1884), T. 2, S. 1843-1846. 
[N] Loew, 0., The physiological rôle of mineral nutrients. Bulletin Nr. 18 of the U. S. Department of agriculture (Division of vegetable physiology and pathology), Washington 1899.

[0] Mendel, L., Brief contributions to physiological chemistry. I. On the occurence of iodine in corals. The americ. journ. of physiology, Bd. IV (1901), S. 243-246.

[P] Milne Edwards, H., Histoire naturelle des corallaires ou polypes proprement dits, T. 1, Paris 1857.

[R] Studer, Th., Versuch eines Systemes der Alcyonaria. Archiv f. Naturgeschichte, Jahrg. 53, Bd. I (1887), S. 1-74.

[S] Valenciennes, M., Extrait d'une monographie de la famille des Gorgonidées de la classe des Polypes. Compt. rend. de l'Académie des sciences, Bd. XLI (1855), S. 7-15.

[T] Vogel, A ug., jun., Über die in den Schwämmen vorkommenden Jodverbindungen. Gelehrte Anzeigen der K. Bayer. Akademie der Wissenschaften, Bd. XXVII (1848), S. 223-227.

[U] Wheeler, H., und Jamieson, G., Synthesis of iodogorcic acid. Americ. chemic. journ., Bd. XXXIII (1905), S. 365-372. 